Bull. Korean Math. Soc. 50 (2013), No. 4, pp. 1243-1259

http://dx.doi.org/10.4134/BKMS.2013.50.4.1243

\title{
ON SOME BEHAVIOR OF INTEGRAL POINTS ON A HYPERBOLA
}

\author{
YEONOK KIM
}

\begin{abstract}
In this paper, we study the root system of rank 2 hyperbolic Kac-Moody algebras. We give some sufficient conditions for the existence of imaginary roots of square length $-2 k\left(k \in \mathbb{Z}_{>0}\right)$. We also give several relations between the integral points on the hyperbola $\mathfrak{h}$ to show that the value of the symmetric bilinear form of any two integral points depends only on the number of integral points between them. We also give some generalizations of Binet formula and Catalan's identity.
\end{abstract}

\section{Introduction}

Let $A$ be the Cartan matrix $A=\left(\begin{array}{cc}2 & -a \\ -b & 2\end{array}\right)$ with $a \geq b>0, a b>4$ and $\mathfrak{g}=\mathfrak{g}(A)$ denote the associated rank 2 hyperbolic Kac-Moody Lie algebra over the field of complex numbers. Let $\Pi=\left\{\alpha_{0}, \alpha_{1}\right\}$ denote the set of simple roots with $\Delta$ its root system, and $W=\left\langle r_{0}, r_{1}\right\rangle$ its Weyl group of $\mathfrak{g}$ where $r_{0}, r_{1}$ are the simple reflections. A root $\alpha \in \Delta$ is called a real root if there exists $w \in W$ such that $w(\alpha)$ is a simple root. A root which is not real is called an imaginary root. We denote by the symbols $\Delta^{r e}, \Delta_{+}^{r e}, \Delta^{i m}$, and $\Delta_{+}^{i m}$ the set of all real, positive real, imaginary and positive imaginary roots, respectively. We also denote by $\Delta_{+, k}^{i m}$ the set of all positive imaginary roots of the algebra $\mathfrak{g}(A)$ with square length $-2 k$. In [2], A. J. Feingold showed that the Fibonacci numbers are intimately related to the rank 2 hyperbolic GCM Lie algebras. Moreover in [5], S. J. Kang and D. J. Melville showed that all the roots of a given length are Weyl conjugate to roots in a small region. These help in determining the sufficient conditions for the existence of integral points on the hyperbola $\mathfrak{h}_{k}: b x^{2}-a b x y+a y^{2}=-b k\left(k \in \mathbb{Z}_{>0}\right)$. Below we display the main results.

Main results. 1. Let $\left\{A_{n}\right\}$ and $\left\{B_{n}\right\}$ be sequences defined in (3). In Lemma 2.2, we give some generalizations of Binet formula for the sequences $\left\{A_{n}\right\}$ and

Received August 21, 2012; Revised February 19, 2013.

2010 Mathematics Subject Classification. 17B67, 17B65, 11B39.

Key words and phrases. Kac-Moody algebra, hyperbolic type, integral point, Binet formula, Catalan's identity. 
$\left\{B_{n}\right\}$. And then we also give binomial expression for $n$-th term of each sequence by utilizing Pascal's triangle.

2. Let $b x^{2}-a b x y+a y^{2}=-b k$ be the hyperbola given above and let $k=t \gamma^{2}$ be any positive integer where $t$ is a square-free integer and $\gamma \in \mathbb{Z}$. For some positive integer $\delta$, we have:

$$
\begin{array}{r}
\text { If }(\gamma, \delta) \in-\check{C} \text {, then } \frac{a b-4}{a b} \leq t \leq \frac{a b-4}{4} \text {, where }-\check{C} \text { is a set defined as } \\
-\check{C}=\left\{(m, n) \in \mathbb{Z}_{\geq 0} \times \mathbb{Z}_{\geq 0} \mid \frac{2 \sqrt{k}}{\sqrt{a b-4}} \leq m \leq \sqrt{\frac{a b k}{a b-4}}\right. \\
\left.n=\frac{a b m-\sqrt{\left(a^{2} b^{2}-4 a b\right) m^{2}-4 a b k}}{2 a}\right\} .
\end{array}
$$

3. $(\gamma, \delta) \in-\check{C}$ if and only if there exists $j \in T_{a, b}$ such that $t=j-\frac{j^{2}}{a b}-1$ and $n=\frac{j m}{a}$ where $T_{a, b}$ is a set defined as

$$
T_{a, b}=\left\{j \in \mathbb{Z} \mid \frac{j^{2}}{a b} \in \mathbb{Z}, \quad \frac{j m}{a} \in \mathbb{Z} \text { with } 2 \leq j \leq \frac{a b}{2}\right\} .
$$

4. Let $b x^{2}-a b x y+a y^{2}=a$ and $b x^{2}-a b x y+a y^{2}=b$ be hyperbolas. Then the value of the symmetric bilinear form (see equation (2) in Section 2) of two integral points depends only on the number of integral points between them.

5. We give some generalizations of Catalan's identity in [6] for the sequences $\left\{A_{n}\right\}$ and $\left\{B_{n}\right\}$ as follows:

(a) $\left(A_{2 n+2 k}\right)^{2}-A_{2 n} A_{2 n+4 k}=\frac{a^{2}}{\alpha^{4 k-4}}\left(\sum_{l=1}^{k} \alpha^{4 k-4 l}\right)^{2}$.

(b) $\left(B_{2 n+2 k}\right)^{2}-B_{2 n} B_{2 n+4 k}=\frac{b^{2}}{\alpha^{4 k-4}}\left(\sum_{l=1}^{k} \alpha^{4 k-4 l}\right)^{2}$.

(c) $\left(A_{2 n+1+2 k}\right)^{2}-A_{2 n+1} A_{2 n+1+4 k}=\frac{a b}{\alpha^{4 k-4}}\left(\sum_{l=1}^{k} \alpha^{4 k-4 l}\right)^{2}$.

And then we define the new sequence $\left\{F_{n}^{(a)}\right\}$, which we will call Lie-Fibonacci sequence, that is a generalization of Fibonacci sequence. We also give several interesting identities for Lie-Fibonacci sequence $\left\{F_{n}^{(a)}\right\}$.

\section{The root system of $\mathfrak{g}(A)$}

In this section, we give some sufficient conditions for the existence of imaginary roots of square length $-2 k(k \in \mathbb{Z}>0)$.

We identify an element

$$
\alpha=x_{0} \alpha_{0}+x_{1} \alpha_{1} \in Q \text { with an ordered pair }\left(x_{0}, x_{1}\right) \in \mathbb{Z} \times \mathbb{Z} .
$$

We call a root $\alpha \in \mathbb{Z} \times \mathbb{Z}$ a positive integral point if $x_{0}, x_{1} \in \mathbb{Z} \geq 0$. Let us define a symmetric bilinear form $(\cdot \mid \cdot)$ on $\mathfrak{h}^{*}$ by the following equations:

$$
\left(\alpha_{0} \mid \alpha_{0}\right)=2, \quad\left(\alpha_{1} \mid \alpha_{1}\right)=\frac{2 a}{b}, \quad\left(\alpha_{0} \mid \alpha_{1}\right)=-a .
$$

We define the sequences of integers $\left\{A_{n}\right\}$ and $\left\{B_{n}\right\}$ for $n \geq 0$ by the recurrence relations

(3) $A_{0}=B_{0}=0, A_{1}=B_{1}=1, r_{0}\left(A_{n} \alpha_{0}+B_{n+1} \alpha_{1}\right)=A_{n+2} \alpha_{0}+B_{n+1} \alpha_{1}$ 
and $\quad r_{1}\left(A_{n+1} \alpha_{0}+B_{n} \alpha_{1}\right)=A_{n+1} \alpha_{0}+B_{n+2} \alpha_{1}$.

Then we obtain the following formulas for $n \geq 0$ :

$$
\begin{gathered}
A_{n+2}=a B_{n+1}-A_{n}, \\
B_{n+2}=b A_{n+1}-B_{n} .
\end{gathered}
$$

In particular, if $a=b=3$, then $A_{n}=B_{n}=F_{2 n}$ for $n \geq 1$, where $\left\{F_{n}\right\}$ is a famous Fibonacci sequence defined by the recurrence relations

$$
F_{0}=0, F_{1}=1, F_{n+2}=F_{n}+F_{n+1} .
$$

Now we recall some well known properties of the root system of hyperbolic Kac-Moody algebra $\mathfrak{g}(A)$.

Proposition 2.1 ([4]). For the hyperbolic Kac-Moody algebra $\mathfrak{g}(A)$, the root system is given by

(a) $\Delta^{r e}=\{(x, y) \in \mathbb{Z} \times \mathbb{Z}) \mid b x^{2}-a b x y+a y^{2}=a$ or $\left.b\right\}$.

(b) $\left.\Delta^{i m}=\{(x, y) \in \mathbb{Z} \times \mathbb{Z}) \mid b x^{2}-a b x y+a y^{2} \leq 0\right\}$.

We know that the Fibonacci numbers can be calculated directly with Binet formula (see [3] and [7]):

$$
F_{n}=\frac{1}{\sqrt{5}}\left(\left(\frac{1+\sqrt{5}}{2}\right)^{n}-\left(\frac{1-\sqrt{5}}{2}\right)^{n}\right) .
$$

The following lemma gives some generalization of Binet formula corresponding to the sequences $\left\{A_{n}\right\}$ and $\left\{B_{n}\right\}$.

Lemma 2.2. Let $\left\{A_{n}\right\}$ and $\left\{B_{n}\right\}$ be the sequences defined in (3). Then

$$
\begin{aligned}
A_{2 n} & =\frac{a \alpha^{2}}{\alpha^{4}-1}\left(\alpha^{2 n}-\frac{1}{\alpha^{2 n}}\right), \\
B_{2 n} & =\frac{b \alpha^{2}}{\alpha^{4}-1}\left(\alpha^{2 n}-\frac{1}{\alpha^{2 n}}\right) \\
A_{2 n+1} & =B_{2 n+1}=\frac{\alpha+\alpha^{3}}{\alpha^{4}-1}\left(\alpha^{2 n+1}-\frac{1}{\alpha^{2 n+1}}\right) \quad\left(n \in \mathbb{Z}_{>0}\right),
\end{aligned}
$$

where

$$
\alpha=\frac{\sqrt{a b}+\sqrt{a b-4}}{2} \quad \text { is a zero of } 1-(a b-2) x^{2}+x^{4} .
$$

Proof. We will look for the closed form expressions of the sequences $\left\{A_{n}\right\}$ and $\left\{B_{n}\right\}$. Let us consider the sequence $\left\{B_{n}\right\}$ first. Let $F(x)=\sum_{n=1}^{\infty} B_{n} x^{n}$ be the generating function for the sequence $\left\{B_{n}\right\}_{n \geq 1}$.

Using the equation (4), we have

$$
B_{n+3}=(a b-2) B_{n+1}-B_{n-1} .
$$

Then multiplying (6) by $x^{n+3}$ yields

$$
B_{n+3} x^{n+3}=(a b-2) x^{2} B_{n+1} x^{n+1}-x^{4} B_{n-1} x^{n-1} .
$$


Summing up over $n \geq 1$ gives

$$
\sum_{n=1}^{\infty} B_{n+3} x^{n+3}=(a b-2) x^{2} \sum_{n=1}^{\infty} B_{n+1} x^{n+1}-x^{4} \sum_{n=1}^{\infty} B_{n-1} x^{n-1} .
$$

These implies,

$$
F(x)-\left(B_{0}+B_{1} x+B_{2} x^{2}+B_{3} x^{3}\right)=(a b-2) x^{2}(F(x)-x)-x^{4} F(x),
$$

and hence

$$
\begin{aligned}
\left(1-(a b-2) x^{2}+x^{4}\right) F(x) & =x+b x^{2}+(a b-1) x^{3}-(a b-2) x^{3} \\
& =x+b x^{2}+x^{3} .
\end{aligned}
$$

Now suppose

$$
\alpha=\frac{\sqrt{a b}+\sqrt{a b-4}}{2}
$$

be a zero of $1-(a b-2) x^{2}+x^{4}$. By brief calculation, we get four zeros, $\alpha,-\alpha$, $\frac{1}{\alpha}$ and $-\frac{1}{\alpha}$, of $1-(a b-2) x^{2}+x^{4}$. Thus we have:

$$
\begin{aligned}
F(x)= & \frac{x+b x^{2}+x^{3}}{1-(a b-2) x^{2}+x^{4}} \\
= & \frac{\alpha+b \alpha^{2}+\alpha^{3}}{2\left(\alpha^{4}-1\right)}\left(\sum_{n=0}^{\infty}(\alpha x)^{n}-\sum_{n=0}^{\infty}\left(\frac{x}{\alpha}\right)^{n}\right) \\
& -\frac{\alpha-b \alpha^{2}+\alpha^{3}}{2\left(\alpha^{4}-1\right)}\left(\sum_{n=0}^{\infty}(-\alpha x)^{n}-\sum_{n=0}^{\infty}\left(-\frac{x}{\alpha}\right)^{n}\right) \\
= & \frac{b \alpha^{2}}{\left(\alpha^{4}-1\right)}\left(\sum_{n=0}^{\infty}(\alpha x)^{2 n}-\sum_{n=0}^{\infty}\left(\frac{x}{\alpha}\right)^{2 n}\right) \\
& +\frac{\alpha+\alpha^{3}}{\left(\alpha^{4}-1\right)}\left(\sum_{n=0}^{\infty}(\alpha x)^{2 n+1}-\sum_{n=0}^{\infty}\left(\frac{x}{\alpha}\right)^{2 n+1}\right) \\
= & \frac{b \alpha^{2}}{\alpha^{4}-1} \sum_{n=0}^{\infty}\left(\alpha^{2 n}-\frac{1}{\alpha^{2 n}}\right) x^{2 n}+\frac{\alpha+\alpha^{3}}{\alpha^{4}-1} \sum_{n=0}^{\infty}\left(\alpha^{2 n+1}-\frac{1}{\alpha^{2 n+1}}\right) x^{2 n+1} .
\end{aligned}
$$

Therefore, we obtain

$$
B_{2 n}=\frac{b \alpha^{2}}{\alpha^{4}-1}\left(\alpha^{2 n}-\frac{1}{\alpha^{2 n}}\right), \quad B_{2 n+1}=\frac{\alpha+\alpha^{3}}{\alpha^{4}-1}\left(\alpha^{2 n+1}-\frac{1}{\alpha^{2 n+1}}\right)(n \geq 0) .
$$

By using similar steps, we have

$$
\begin{gathered}
A_{2 n}=\frac{a \alpha^{2}}{\alpha^{4}-1}\left(\alpha^{2 n}-\frac{1}{\alpha^{2 n}}\right), \\
A_{2 n+1}=B_{2 n+1}=\frac{\alpha+\alpha^{3}}{\alpha^{4}-1}\left(\alpha^{2 n+1}-\frac{1}{\alpha^{2 n+1}}\right) \quad(n \geq 1),
\end{gathered}
$$

and hence the stated result. 
Using the above lemma, we now obtain a binomial expression for each of the sequences $\left\{A_{n}\right\}$ and $\left\{B_{n}\right\}$.

Theorem 2.3. Let $\left\{A_{n}\right\}$ and $\left\{B_{n}\right\}$ be the sequences as above. Then for all $n \geq 0$,

$$
\begin{aligned}
& A_{2 n}=\frac{a}{2^{2 n-1}} \sum_{k=1}^{n} 2 n C_{2 k-1}(a b)^{n-k}(a b-4)^{k-1}, \\
& B_{2 n}=\frac{b}{2^{2 n-1}} \sum_{k=1}^{n} 2{ }_{2 n} C_{2 k-1}(a b)^{n-k}(a b-4)^{k-1},
\end{aligned}
$$

and

$$
A_{2 n+1}=B_{2 n+1}=\frac{1}{2^{2 n}} \sum_{k=1}^{n+1} 2 n+1 C_{2 k-1} a b^{n+1-k}(a b-4)^{k-1} .
$$

Proof. From Lemma 2.2,

$$
\begin{aligned}
B_{2 n} & =\frac{b \alpha^{2}}{\alpha^{4}-1}\left(\alpha^{2 n}-\frac{1}{\alpha^{2 n}}\right) \\
& =\frac{b\left(\frac{\sqrt{a b}+\sqrt{a b-4}}{2}\right)^{2}}{\left(\frac{\sqrt{a b}+\sqrt{a b-4}}{2}\right)^{4}-1}\left(\left(\frac{\sqrt{a b}+\sqrt{a b-4}}{2}\right)^{2 n}-\left(\frac{\sqrt{a b}-\sqrt{a b-4}}{2}\right)^{2 n}\right) \\
& =\frac{b}{\sqrt{a b(a b-4)}}\left(\left(\frac{\sqrt{a b}+\sqrt{a b-4}}{2}\right)^{2 n}-\left(\frac{\sqrt{a b}-\sqrt{a b-4}}{2}\right)^{2 n}\right) \\
& =\frac{b}{2^{2 n-1}} \sum_{k=1}^{n} 2 n C_{2 k-1}(a b)^{n-k}(a b-4)^{k-1}
\end{aligned}
$$

and

$$
\begin{aligned}
B_{2 n+1} & =\frac{\alpha+\alpha^{3}}{\alpha^{4}-1}\left(\alpha^{2 n+1}-\frac{1}{\alpha^{2 n+1}}\right) \\
& =\frac{\frac{\sqrt{a b}+\sqrt{a b-4}}{2}+\left(\frac{\sqrt{a b}+\sqrt{a b-4}}{2}\right)^{3}}{\left(\frac{\sqrt{a b}+\sqrt{a b-4}}{2}\right)^{4}-1}\left(\left(\frac{\sqrt{a b}+\sqrt{a b-4}}{2}\right)^{2 n+1}-\left(\frac{\sqrt{a b}-\sqrt{a b-4}}{2}\right)^{2 n+1}\right) \\
& =\frac{1}{2^{2 n-1}} \sum_{k=1}^{n+1} 2 n+1 C_{2 k-1}(a b)^{n+1-k}(a b-4)^{k-1} .
\end{aligned}
$$

Since $A_{2 n}=\frac{a}{b} B_{2 n}$ and $A_{2 n+1}=B_{2 n+1}$, we get the desired result.

From now on, we can get the $n$-th term of $\left\{A_{n}\right\}$ and $\left\{B_{n}\right\}$ by using the Pascal's triangle. 
Example 2.4. Since

$$
A_{8}=\frac{a}{2^{7}} \sum_{k=1}^{4}{ }_{8} C_{2 k-1}(a b)^{n-k}(a b-4)^{k-1},
$$

in the 8th row of the Pascal's triangle, we take the 2nd 8, 4th 56, 6th 56, and 8 th 8 . Then we have

$$
A_{8}=\frac{a}{2^{7}}\left(8(a b)^{3}+56(a b)^{2}(a b-4)+56(a b)(a b-4)^{2}+8(a b-4)^{3}\right) .
$$

In the same manner, we get

$B_{9}=\frac{a}{2^{8}}\left(9(a b)^{4}+84(a b)^{3}(a b-4)+126(a b)^{2}(a b-4)^{2}+36(a b)(a b-4)^{3}+(a b-4)^{4}\right)$.

So far, we have looked at the expressions of the sequences $\left\{A_{n}\right\}$ and $\left\{B_{n}\right\}$. Now we investigate relations among the integral points on some hyperbola. In particular, we will look at imaginary roots first.

We will start with recalling some of the fundamental properties of the imaginary roots.

Proposition 2.5 ([4]). (a) The set $\Delta_{+}^{i m}$ is $W$-invariant.

(b) For any $\alpha \in \Delta_{+, k}^{i m}$, there exists a unique $\beta \in \Delta_{+}^{i m}$ such that $\beta=w(\alpha)$ for some $w \in W$ and $\left(\beta \mid \alpha_{i}\right) \leq 0$ for $i=0,1$.

For any $\alpha \in \Delta_{+, k}^{i m}$, the above proposition tells us that there is a unique $\beta=(m, n) \in \Delta_{+}^{i m}$ such that $\beta=w \alpha$ for some $w \in W$ and

$$
\begin{gathered}
\left(\beta \mid \alpha_{0}\right)=2 m-a n \leq 0, \\
\left(\beta \mid \alpha_{1}\right)=-a m+\frac{2 a}{b} n \leq 0 .
\end{gathered}
$$

Since the bilinear form $(\mid)$ is $W$-invariant, we also have $\beta \in \Delta_{+, k}^{i m}$.

If $-\check{C}$ is the set of all positive integral points $\beta=(m, n)$ on the hyperbola $\mathfrak{h}_{k}: b x^{2}-a b x y+a y^{2}=-b k\left(k \in \mathbb{Z}_{>0}\right)$ that lie between the lines $y=\frac{2}{a} x$ and $y=\frac{b}{2} x$, then we observe that (see [4] and [5])

$$
\begin{aligned}
-\check{C}=\left\{(m, n) \in \mathbb{Z}_{\geq 0} \times \mathbb{Z}_{\geq 0} \mid\right. & \frac{2 \sqrt{k}}{\sqrt{a b-4}} \leq m \leq \sqrt{\frac{a b k}{a b-4}}, \\
n & \left.=\frac{a b m-\sqrt{\left(a^{2} b^{2}-4 a b\right) m^{2}-4 a b k}}{2 a}\right\} .
\end{aligned}
$$

We have the following proposition.

Proposition 2.6 ([5]). (a) For $\alpha \in \Delta_{+}^{i m}$, there exists a unique $\beta \in-\check{C}$ such that $\alpha=w \beta$ for some $w \in W$.

$$
\begin{aligned}
\text { (b) } \Delta_{+, k}^{i m}= & \left\{(m, n),\left(m A_{2 j+1}-n A_{2 j}, m B_{2 j}-n B_{2 j-1}\right),\right. \\
& \left(-m A_{2 j-1}+n A_{2 j},-m B_{2 j}+n B_{2 j+1}\right), \\
& \left(m A_{2 j+1}-n A_{2 j}, m B_{2 j+2}-n B_{2 j+1}\right), \\
& \left.\left(-m A_{2 j+1}+n A_{2 j+2},-m B_{2 j}+n B_{2 j+1}\right) \mid(m, n) \in-\check{C}\right\} .
\end{aligned}
$$


We know that any positive integer $k$ can be represented as a product of two integers $t$ and $\gamma^{2}(\gamma \in \mathbb{Z})$ where $t$ is a square free integer.

The following lemma gives a nice description on a set of positive imaginary roots of square length $-2 k$.

Lemma 2.7. Let $b x^{2}-a b x y+a y^{2}=-b k$ be a hyperbola where $k=t \gamma^{2}$ is any positive integer with a square free integer $t$ and $\gamma \in \mathbb{Z}_{>0}$. If $(\gamma, \delta) \in-\check{C}$ for some positive integer $\delta$, then

$$
\frac{a b-4}{a b} \leq t \leq \frac{a b-4}{4}
$$

Proof. If $(\gamma, \delta) \in-\check{C}$ for some positive integer $\delta$, then

$$
\frac{2 \sqrt{k}}{\sqrt{a b-4}} \leq \gamma \leq \sqrt{\frac{a b k}{a b-4}} .
$$

Since $k=t \gamma^{2}$, we have

$$
\frac{2 \sqrt{t}}{\sqrt{a b-4}} \leq 1 \leq \sqrt{\frac{a b t}{a b-4}} .
$$

Squaring both sides give the desired result.

We now present the main result of this article in the next theorem.

Theorem 2.8. Let $k=t \gamma^{2}$ be any positive integer, where $t$ is a square free integer and let

$$
T_{a, b}=\left\{j \in \mathbb{Z} \mid \frac{j^{2}}{a b} \in \mathbb{Z}, \quad \frac{j \gamma}{a} \in \mathbb{Z} \text { with } 2 \leq j \leq \frac{a b}{2}\right\} .
$$

Then

$(\gamma, \delta) \in-\check{C}$ for some positive integer $\delta$ if and only if

$$
t=j-\frac{j^{2}}{a b}-1, \quad \text { and } \delta=\frac{j \gamma}{a} \text { for some } j \in T_{a, b} .
$$

Proof. Suppose that $(\gamma, \delta) \in-\check{C}$ for some positive integer $\delta$. From Lemma 2.7, we have $a b t \geq a b-4$. Hence

$$
\begin{aligned}
0 \leq \sqrt{\left(a^{2} b^{2}-4 a b\right) \gamma^{2}-4 a b k} & =\sqrt{\left(a^{2} b^{2}-4 a b-4 a b t\right)} \gamma \\
& \leq \sqrt{a^{2} b^{2}-4 a b-4(a b-4)} \gamma \\
& =(a b-4) \gamma .
\end{aligned}
$$

Since $2 a b+1 \notin 2 \mathbb{Z}$, it is impossible to have

$$
\sqrt{\left(a^{2} b^{2}-4 a b\right)-4 a b t}=a b-(2 j+1) \text { for any } j \text { with } 2 \leq j \leq \frac{a b}{2} .
$$

Thus we have

(9) $\sqrt{\left(a^{2} b^{2}-4 a b\right)-4 a b t} \in \mathbb{Z}$ if and only if $\sqrt{\left(a^{2} b^{2}-4 a b\right)-4 a b t}=a b-2 j$ for some $j$ with $2 \leq j \leq \frac{a b}{2}$. 
Squaring both sides of equation (9), we obtain

$$
t=j-\frac{j^{2}}{a b}-1
$$

and hence

$$
\frac{j^{2}}{a b} \in \mathbb{Z}
$$

On the other hand,

$$
\delta=\left(\frac{a b-\sqrt{a^{2} b^{2}-4 a b-4 a b t}}{2 a}\right) \gamma=\left(\frac{a b-(a b-2 j)}{2}\right) \gamma=\frac{j \gamma}{a} .
$$

Since $\delta$ is a positive integer, we have $j \in T_{a, b}$. Conversely, suppose that there exists $j \in T_{a, b}$ such that

$$
t=j-\frac{j^{2}}{a b}-1 \quad \text { and } \quad \frac{j \gamma}{a} \in \mathbb{Z}_{>0}
$$

Consider the hyperbola $\mathfrak{h}_{t \gamma^{2}}: b x^{2}-a b x y+a y^{2}=-b t \gamma^{2}$. If we replace $(x, y)$ with $\left(\gamma, \frac{j \gamma}{a}\right)$ in the equation $\mathfrak{h}_{t \gamma^{2}}: b x^{2}-a b x y+a y^{2}=-b t \gamma^{2}$, we obtain

$$
b \gamma^{2}-a b \gamma\left(\frac{j \gamma}{a}\right)+a\left(\frac{j \gamma}{a}\right)^{2}=-b\left(j-\frac{j^{2}}{a b}-1\right) \gamma^{2}=-b t \gamma^{2},
$$

which shows that $\left(\gamma, \frac{j \gamma}{a}\right)$ is an integral point on the hyperbola $\mathfrak{h}_{t \gamma^{2}}$. To prove $\left(\gamma, \frac{j \gamma}{a}\right) \in \check{C}$, we need to check that the inequality

$$
\frac{2 \sqrt{k}}{\sqrt{a b-4}} \leq \gamma \leq \sqrt{\frac{a b k}{a b-4}}
$$

and

$$
\frac{j \gamma}{a}=\frac{a b \gamma-\sqrt{\left(a^{2} b^{2}-4 a b\right) \gamma^{2}-4 a b k}}{2 a} .
$$

Since $t=j-\frac{j^{2}}{a b}-1$ implies equation (11), we only need to show the equation (10). We will show instead that

$$
\frac{a b-4}{a b} \leq t \leq \frac{a b-4}{4},
$$

because of the direct equivalence between (10) and (12). It is clear that $\frac{a b-4}{a b} \leq t$ since $t \geq 1$. We only need to show

$$
\frac{a b-4}{4} \leq t
$$

Suppose $t>\frac{a b-4}{4}$. Then we have

and hence

$$
j-\frac{j^{2}}{a b}-1>\frac{a b-4}{4},
$$

$$
(a b-2 j)^{2}<0,
$$


which is a contradiction. Hence the inequality (12) holds and hence

$$
\left(\gamma, \frac{j \gamma}{a}\right) \in-\check{C}
$$

We already know that the Weyl group $W$ is infinite and acts faithfully on the root system of $\mathfrak{g}(A)$. Thus

$$
\Delta_{+}^{i m}=\left\{\alpha \in Q_{+} \mid \alpha=w \beta \text { for } w \in W, \beta \in-\check{C}\right\}
$$

and we can say that $\Delta_{+}^{i m}$ is either empty or infinite. Since there are no integral point on the union of the lines $b x^{2}-a b x y+a y^{2}=0$, the set of all imaginary roots of $\mathfrak{g}(A)$ corresponds to the set of all integral points on the hyperbolas $\mathfrak{h}_{k}: b x^{2}-a b x y+a y^{2}=-b k\left(k \in \mathbb{Z}_{>0}\right)($ see $[4]$ and $[5])$.

We have the following corollary:

Corollary 2.9. If $T_{a, b} \neq \emptyset$, for some positive integer $\gamma$, then there are infinitely many integral points on the hyperbola

$$
\mathfrak{h}_{\left(j-\frac{j^{2}}{a b}-1\right) \gamma^{2}}: b x^{2}-a b x y+a y^{2}=-b\left(j-\frac{j^{2}}{a b}-1\right) \gamma^{2} \text { for } j \in T .
$$

Proof. If there exists $j \in T_{a, b}$, then $\frac{j \gamma}{a} \in \mathbb{Z}$. On the other hand,

$$
\begin{aligned}
b \gamma^{2}-a b \gamma\left(\frac{j \gamma}{a}\right)+a\left(\frac{j}{a}\right)^{2} & =b \gamma^{2}-b j \gamma^{2}+b\left(\frac{j^{2} \gamma^{2}}{a b}\right) \\
& =-b\left(j-\frac{j^{2}}{a b}-1\right) \gamma^{2}
\end{aligned}
$$

This means that $\left(\gamma, \frac{j \gamma}{a}\right)$ is an integral point on the hyperbola $\mathfrak{h}_{\left(j-\frac{j^{2}}{a b}-1\right) \gamma^{2}}$. Since $W$ is infinite and $W$ acts faithfully on $\Delta^{i m}$, we have the desired result.

The above corollary tells us that if $j \in T_{a, b}=\emptyset$, then $-\check{C}=\emptyset$. The following example, however, shows that the converse is not valid.

Example 2.10. Let $2 x^{2}-10 x y+5 y^{2}=-12$. Since $(2,2) \in-\check{C}$, there exist infinitely many integral points on the hyperbola. But $T_{a, b}=\emptyset$.

The following theorem gives us a useful algorithm for finding all of $j \in T_{a, b}$.

Theorem 2.11. Suppose $a b=u p^{2}$ where $u$ is a square-free integer and $p$ is a positive integer and let $j_{0}=u p$. Then $j \in T_{a, b}$ if and only if

$$
j=q j_{0} \text { for some positive integer } q \text { with } 1 \leq q \leq \frac{\frac{a b}{2}}{j_{0}} \text { and } \frac{j m}{a} \in \mathbb{Z}_{>0} .
$$

Proof. Assume $j \in T_{a, b}$. Then $j^{2}=l a b=l u p^{2}$ for some $l \in \mathbb{Z}_{>0}$. Since $u$ is a square free integer, we have $l=u q^{2}$ for some $q \in \mathbb{Z}_{>0}$.

Thus

Moreover since

$$
j=q u p=q j_{0}
$$

$$
2 \leq j=q j_{0} \leq \frac{a b}{2}, \text { and } j_{0} \geq 2,
$$


we have

$$
1 \leq q \leq \frac{\frac{a b}{2}}{j_{0}},
$$

the desired result. Conversely, suppose that

$$
j=q j_{0} \text { for some } q \text { with } 1 \leq q \leq \frac{\frac{a b}{2}}{j_{0}} \text { and } \frac{j m}{a} \in \mathbb{Z}_{>0} .
$$

Since $j_{0} \geq 2$, we have $2 \leq j \leq \frac{a b}{2}$. On the other hand, $\frac{j^{2}}{a b}=\frac{\left(q j_{0}\right)^{2}}{a b}=$ $\frac{q^{2} u^{2} p^{2}}{a b}=q^{2} u \in \mathbb{Z}$. Thus we have $j \in T_{a, b}$, establishing the statement.

Corollary 2.12. If $a b$ is a square-free integer, then $T_{a, b}=\emptyset$.

Proof. Since $j_{0}=a b$, there cannot exist $q \in \mathbb{Z}$ such that $q j_{0} \leq \frac{a b}{2}$.

We present several examples.

Example 2.13. Let $\mathfrak{h}_{k}: 4 x^{2}-36 x y+9 y^{2}=-4 k$ where $k=7 \cdot 3^{2}$. Then $T_{9,4}=\{6,12,18\}$. Since $j-\frac{j^{2}}{36}-1=7$, we have $j=12 \in T_{9,4}$, and $\left(\gamma \cdot \frac{j \gamma}{a}\right)=$ $(3,4) \in-\check{C}$. Hence there are infinitely many integral points on the hyperbola $4 x^{2}-36 x y+9 y^{2}=-4 \cdot 7 \cdot 3^{2}$.

From our discussion, it is clear that $-\check{C}=\emptyset$ if and only if there is no integral points on the hyperbola $\mathfrak{h}_{k}: b x^{2}-a b x y+a y^{2}=-b k\left(k \in \mathbb{Z}_{>0}\right)$. Nevertheless, it is not the same way as in the case of $T_{a, b}=\emptyset$. The following example is the case when $-\check{C}=\emptyset$.

Example 2.14. There is no integral point on the hyperbola $2 x^{2}-10 x y+5 y^{2}=$ $-2$

Example 2.15. Since $(2,2)$ is an integral point on the hyperbola $2 x^{2}-10 x y+$ $5 y^{2}=-12$, there are infinitely many integral points. But $T_{a, b}=\emptyset$.

\section{Some relations between the integral points on the hyperbolas}

In this section, we study some relations among the integral points on the hyperbolas $b x^{2}-a b x y+a y^{2}=b$ or $a$. In the case of $a \neq b r^{2}$ for some $r \in \mathbb{Z}_{+}$, it is well known that there is an 1-1 correspondence between the set $\Delta^{r e}$ and the set of all integral points on the hyperbolas $\mathfrak{h}^{a}: b x^{2}-a b x y+a y^{2}=a$ and $\mathfrak{h}^{b}: b x^{2}-a b x y+a y^{2}=b$.

Using induction on $j \geq 0$, we have the following equations:

$$
\begin{gathered}
\left(r_{0} r_{1}\right)^{j} \alpha_{0}=\left(A_{2 j+1}, B_{2 j}\right), \\
\left(r_{1} r_{0}\right)^{j} r_{1} \alpha_{0}=\left(A_{2 j+1}, B_{2 j+2}\right), \\
\left(r_{1} r_{0}\right)^{j} \alpha_{1}=\left(A_{2 j}, B_{2 j+1}\right), \text { and } \\
r_{0}\left(r_{1} r_{0}\right)^{j} \alpha_{1}=\left(A_{2 j+2}, B_{2 j+1}\right) .
\end{gathered}
$$

Consider the hyperbolas $\mathfrak{h}^{a}$ and $\mathfrak{h}^{b}$ as above. It consists of two pairs of curves. Let us classify $\Delta_{+}^{r e}$ into four groups. By simple calculation, for $j \in \mathbb{Z}_{>0}$, we have the following: 
(a) The point $\left(A_{2 j}, B_{2 j+1}\right)$ is an integral point on the hyperbola $\mathfrak{h}^{a}$ that lies above the asymptote $y=\left(\frac{a b+\sqrt{a b(a b-4)}}{2 a}\right) x$.

(b) The point $\left(A_{2 j+2}, B_{2 j+1}\right)$ is an integral point on the hyperbola $\mathfrak{h}^{a}$ that lies below the asymptote $y=\left(\frac{a b-\sqrt{a b(a b-4)}}{2 a}\right) x$.

(c) The point $\left(A_{2 j+1}, B_{2 j+2}\right)$ is an integral point on the hyperbola $\mathfrak{h}^{b}$ that lies above the asymptote $y=\left(\frac{a b+\sqrt{a b(a b-4)}}{2 a}\right) x$.

(d) The point $\left(A_{2 j+1}, B_{2 j}\right)$ is an integral point on the hyperbola $\mathfrak{h}^{b}$ that lies below the asymptote $y=\left(\frac{a b-\sqrt{a b(a b-4)}}{2 a}\right) x$.

Since the sequences $\left\{A_{2 n}\right\},\left\{B_{2 n}\right\}$ and $\left\{A_{2 n+1}\right\}$ are increasing, we recognize that there are $n-1$ integral points on the hyperbola between the points $\left(A_{j}, B_{j+1}\right)$ and $\left(A_{j+2 n}, B_{j+2 n+1}\right)$ along the curve. Likewise, there are $n-1$ integral points on the hyperbola between the points $\left(A_{j}, B_{j-1}\right)$ and $\left(A_{j+2 n}, B_{j+2 n-1}\right)$ along the curve.

As mentioned in the introduction, the following theorem shows that the value of the symmetric bilinear form (see equation (2) of Section 2) of two integral points depends only on the number of integral points that lie between them.

Theorem 3.1. Let $\left\{A_{n}\right\}$ and $\left\{B_{n}\right\}$ be sequences defined as in (3) of Section 2. Then for $j, n \in \mathbb{Z}_{\geq 0}$, we have:

(a) $\left(\left(A_{2 j}, B_{2 j+1}\right) \mid\left(A_{2 j+2 n}, B_{2 j+2 n+1}\right)\right)=-a A_{2 n}+\frac{2 a}{b} B_{2 n+1}$.

(b) $\left(\left(A_{2 j+2}, B_{2 j+1}\right) \mid\left(A_{2 j+2 n+2}, B_{2 j+2 n+1}\right)\right)=-a A_{2 n}+\frac{2 a}{b} B_{2 n+1}$.

(c) $\left(\left(A_{2 j+1}, B_{2 j+2}\right) \mid\left(A_{2 j+2 n+1}, B_{2 j+2 n+2}\right)\right)=2 A_{2 n+1}-a B_{2 n}$.

(d) $\left(\left(A_{2 j+1}, B_{2 j}\right) \mid\left(A_{2 j+2 n+1}, B_{2 j+2 n}\right)\right)=2 A_{2 n+1}-a B_{2 n}$.

(e) $\left(\left(A_{2 j}, B_{2 j+1}\right) \mid\left(A_{2 j+2 n+1}, B_{2 j+2 n+2}\right)\right)=-a A_{2 n+1}+\frac{2 a}{b} B_{2 n+2}$.

(f) $\left(\left(A_{2 j+1}, B_{2 j+2}\right) \mid\left(A_{2 j+2 n+2}, B_{2 j+2 n+3}\right)\right)=2 A_{2 n+2}-a B_{2 n+1}$.

Proof. Using the equations in (13), we have

$$
\begin{aligned}
\left(\left(A_{2 j}, B_{2 j+1}\right) \mid\left(A_{2 j+2 n}, B_{2 j+2 n+1}\right)\right) & \left.=\left(r_{1} r_{0}\right)^{j} \alpha_{1} \mid\left(r_{1} r_{0}\right)^{j+n} \alpha_{1}\right) \\
& =\left(\alpha_{1} \mid\left(r_{1} r_{0}\right)^{n} \alpha_{1}\right) \\
& =\left(\alpha_{1} \mid\left(A_{2 n}, B_{2 n+1}\right)\right) \\
& =-a A_{2 n}+\frac{2 a}{b} B_{2 n+1},
\end{aligned}
$$

and hence (a). For (b),

$$
\begin{aligned}
\left(\left(A_{2 j+2}, B_{2 j+1}\right) \mid\left(A_{2 j+2 n+2}, B_{2 j+2 n+1}\right)\right) & =\left(r_{0}\left(r_{1} r_{0}\right)^{j} \alpha_{1} \mid r_{0}\left(r_{1} r_{0}\right)^{j+n} \alpha_{1}\right) \\
& =\left(\alpha_{1} \mid\left(r_{1} r_{0}\right)^{n} \alpha_{1}\right) \\
& =\left(\alpha_{1} \mid\left(A_{2 n}, B_{2 n+1}\right)\right) \\
& =-a A_{2 n}+\frac{2 a}{b} B_{2 n+1},
\end{aligned}
$$


and for $(\mathrm{c})$,

$$
\begin{aligned}
\left(\left(A_{2 j+1}, B_{2 j+2}\right) \mid\left(A_{2 j+2 n+1}, B_{2 j+2 n+2}\right)\right) & =\left(r_{1}\left(r_{0} r_{1}\right)^{j} \alpha_{0} \mid\left(r_{1}\left(r_{0} r_{1}\right)^{j+n} \alpha_{0}\right)\right. \\
& =\left(\alpha_{0} \mid\left(r_{0} r_{1}\right)^{n} \alpha_{0}\right) \\
& =\left(\alpha_{0} \mid\left(A_{2 n+1}, B_{2 n}\right)\right) \\
& =2 A_{2 n+1}-a B_{2 n} .
\end{aligned}
$$

In a similar manner, we can derive the part (d), (e) and (f).

We already know that Catalan's identity (see [6]) which was proved by Catalan holds for Fibonacci sequence $\left\{F_{n}\right\}$. The following properties are some generalizations of Catalan's identity .

Theorem 3.2. (a) $\left(A_{2 n+2 k}\right)^{2}-A_{2 n} A_{2 n+4 k}=\frac{a^{2}}{\alpha^{4 k-4}}\left(\sum_{l=1}^{k} \alpha^{4 k-4 l}\right)^{2}$.

(b) $\left(B_{2 n+2 k}\right)^{2}-B_{2 n} B_{2 n+4 k}=\frac{b^{2}}{\alpha^{4 k-4}}\left(\sum_{l=1}^{k} \alpha^{4 k-4 l}\right)^{2}$.

(c) $\left(A_{2 n+1+2 k}\right)^{2}-A_{2 n+1} A_{2 n+1+4 k}=\frac{a b}{\alpha^{4 k-4}}\left(\sum_{l=1}^{k} \alpha^{4 k-4 l}\right)^{2}$.

Proof. Using Lemma 2.2, we have

$$
\begin{aligned}
& \left(A_{2 n+2 k}\right)^{2}-A_{2 n} A_{2 n+4 k} \\
= & \left(\frac{a \alpha^{2}}{\alpha^{4}-1}\right)^{2}\left\{\left(\alpha^{2 n+2 k}-\frac{1}{\alpha^{2 n+2 k}}\right)^{2}-\left(\alpha^{2 n}-\frac{1}{\alpha^{2 n}}\right)\left(\alpha^{2 n+4 k}-\frac{1}{\alpha^{2 n+4 k}}\right)\right\} \\
= & \left(\frac{a \alpha^{2}}{\alpha^{4}-1}\right)^{2}\left\{\alpha^{4 n+4 k}-\frac{1}{\alpha^{4 n+4 k}}-2-\left(\alpha^{4 n+4 k}-\frac{1}{\alpha^{4 n+4 k}}-\alpha^{4 k}-\frac{1}{\alpha^{4 k}}\right)\right\} \\
= & \frac{a^{2}}{\alpha^{4 k-4}}\left(\frac{\alpha^{4 k}-1}{\alpha^{4}-1}\right)^{2} \\
= & \frac{a^{2}}{\alpha^{4 k-4}}\left(\sum_{l=1}^{k} \alpha^{4 k-4 l}\right)^{2},
\end{aligned}
$$

and hence (a). In a similar manner, we can derive the parts (b) and (c).

It is interesting to see that when we put $k=1$, in Theorem 3.2 , we get the following numbers:

Corollary 3.3. (a) $\left(A_{2 n+2}\right)^{2}-A_{2 n} A_{2 n+4}=a^{2}$.

(b) $\left(B_{2 n+2}\right)^{2}-B_{2 n} B_{2 n+4}=b^{2}$.

(c) $\left(A_{2 n+3}\right)^{2}-A_{2 n+1} A_{2 n+5}=a b$.

Define a new sequence $\left\{F_{n}^{(a)}\right\}$ by the recurrence relations

$$
\begin{aligned}
& F_{0}^{(a)}=F_{1}^{(a)}=1, \quad F_{2 n+2}^{(a)}=a F_{2 n}^{(a)}-F_{2 n-2}^{(a)}, \\
& F_{2 n+1}^{(a)}=F_{2 n+2}^{(a)}-F_{2 n}^{(a)} \quad(n>0) .
\end{aligned}
$$

Clearly, $\left\{F_{n}^{(3)}\right\}=\left\{F_{n}\right\}$, Fibonacci sequence. We deduce, after little calculation, the following proposition. 
Lemma 3.4. For any positive integer $n$ and $\gamma$, we have

(a) $\gamma\left(F_{2 n-2}^{(a)}, F_{2 n}^{(a)}\right)$ is an integral point on the hyperbola $x^{2}-a x y+y^{2}=\gamma^{2}$.

(b) $\gamma\left(F_{2 n-1}^{(a)}, F_{2 n+1}^{(a)}\right)$ is an integral point on the hyperbola $x^{2}-a x y+y^{2}=$ $-(a-2) \gamma^{2}$.

(c) $F_{2 n+3}=a F_{2 n+1}-F_{2 n-1}$.

(d) $F_{2 n}+F_{2 n+1}=F_{2 n+2}$.

(e) $F_{2 n-1}+a F_{2 n}=F_{2 n+1}$.

Proof. By putting $\gamma\left(F_{2 n-2}^{(a)}, F_{2 n}^{(a)}\right)$ and $\gamma\left(F_{2 n-1}^{(a)}, F_{2 n+1}^{(a)}\right)$ instead of $(x, y)$ in the equations $x^{2}-a x y+y^{2}=\gamma^{2}$ and $x^{2}-a x y+y^{2}=-(a-2) \gamma^{2}$, we derive (a) and (b). For (c),

$$
\begin{aligned}
F_{2 n+3}^{(a)} & =F_{2 n+4}^{(a)}-F_{2 n+2}^{(a)} \\
& =a\left(F_{2 n+2}^{(a)}-F_{2 n}^{(a)}\right)-\left(a F_{2 n}^{(a)}-F_{2 n-2}^{(a)}\right) \\
& =a\left(F_{2 n+2}^{(a)}-F_{2 n}^{(a)}\right)-\left(F_{2 n}^{(a)}-F_{2 n-2}^{(a)}\right) \\
& =a F_{2 n+1}^{(a)}-F_{2 n-1}^{(a)} .
\end{aligned}
$$

In a similar manner, we can derive parts (d) and (e).

Let $\alpha=\frac{a+\sqrt{a^{2}-4}}{2}$ be a zero of $1-\left(a^{2}-2\right) x^{2}+x^{4}$. We have the following proposition.

\section{Proposition 3.5.}

(a)

$$
F_{2 n}^{(a)}=\frac{1}{(a-2)^{n} \sqrt{a^{2}-4}}\left(\left(\frac{a-2+\sqrt{a^{2}-4}}{2}\right)^{2 n}-\left(\frac{a-2-\sqrt{a^{2}-4}}{2}\right)^{2 n}\right) .
$$

(b)

$$
F_{2 n+1}^{(a)}=\frac{1}{(a-2)^{n} \sqrt{a^{2}-4}}\left(\left(\frac{a-2+\sqrt{a^{2}-4}}{2}\right)^{2 n+1}-\left(\frac{a-2-\sqrt{a^{2}-4}}{2}\right)^{2 n+1}\right) .
$$

Proof. In case of $a=b$, we derive $\alpha^{2}+1=a \alpha$ and $(\alpha-1)^{2}=(a-2) \alpha$ where $\alpha=\frac{a+\sqrt{a^{2}-4}}{2}$. From Lemma 2.2, we have

$$
\begin{aligned}
F_{2 n}^{(a)} & =\frac{a \alpha^{2}}{\alpha^{4}-1}\left(\alpha^{n}-\frac{1}{\alpha^{n}}\right) \\
& =\frac{1}{\sqrt{a^{2}-4}}\left(\left(\frac{(\alpha-1)^{2}}{a-2}\right)^{n}-\left(\frac{\left(\frac{1}{\alpha}-1\right)^{2}}{a-2}\right)\right) \\
& =\frac{1}{(a-2)^{n} \sqrt{a^{2}-4}}\left((\alpha-1)^{2 n}-\left(\frac{1}{\alpha}-1\right)^{2 n}\right)
\end{aligned}
$$




$$
=\frac{1}{(a-2)^{n} \sqrt{a^{2}-4}}\left(\left(\frac{a-2+\sqrt{a^{2}-4}}{2}\right)^{2 n}-\left(\frac{a-2-\sqrt{a^{2}-4}}{2}\right)^{2 n}\right),
$$

and hence (a). For (b),

$$
\begin{aligned}
F_{2 n+1}^{(a)} & =F^{(a)} 2 n+2-F^{(a)} 2 n \\
& =\frac{a \alpha^{2}}{\alpha^{4}-1}\left(\left(\alpha^{n+1}-\frac{1}{\alpha^{n+1}}\right)-\left(\alpha^{n}-\frac{1}{\alpha^{n}}\right)\right) \\
& =\frac{1}{\sqrt{a^{2}-4}}\left(\alpha^{n}(\alpha-1)-\left(\frac{1}{\alpha}\right)^{n}\left(\frac{1}{\alpha}-1\right)\right) \\
& =\frac{1}{(a-2)^{n} \sqrt{a^{2}-4}}\left((\alpha-1)^{2 n+1}-\left(\frac{1}{\alpha}-1\right)^{2 n+1}\right) \\
& =\frac{1}{(a-2)^{n} \sqrt{a^{2}-4}}\left(\left(\frac{a-2+\sqrt{a^{2}-4}}{2}\right)^{2 n+1}-\left(\frac{a-2-\sqrt{a^{2}-4}}{2}\right)^{2 n+1}\right) .
\end{aligned}
$$

Recall that $\sum_{i=2}^{2 n} F_{i} F_{i-1}^{(a)}=\left(F_{2 n}\right)^{2}$, and $(a-2) \sum_{i=2}^{2 n+1} F_{i} F_{i-1}^{(a)}=\left(F_{2 n+1}\right)^{2}-1$ (see [1]).

The following proposition shows a similar relation between the area of certain rectangle and the partial sum of sequence $\left\{F_{n}^{a} F_{n-1}^{a}\right\}$ of product of two consecutive terms of Lie-Fibonacci sequence $\left\{F_{n}^{(a)}\right\}$.

Proposition 3.6. (a) $\sum_{i=2}^{2 n} F_{i}^{(a)} F_{i-1}^{(a)}=\left(F_{2 n}^{(a)}\right)^{2}$.

(b) $(a-2) \sum_{i=2}^{2 n+1} F_{i}^{(a)} F_{i-1}^{(a)}=\left(F_{2 n+1}^{(a)}\right)^{2}-1$.

Proof. Since

$$
\sum_{i=2}^{2} F_{i}^{(a)} F_{i-1}^{(a)}=F_{2}^{(a)} F_{1}^{(a)}=\left(F_{2}^{(a)}\right)^{2}
$$

(a) is obviously true for $n=1$. Proceeding by induction on $n$, if it is assumed true for $n=k$, then

$$
\begin{aligned}
\sum_{i=2}^{2 k+2} F_{i}^{(a)} F_{i-1}^{(a)} & =\sum_{i=2}^{2 k} F_{i}^{(a)} F_{i-1}^{(a)}+F_{2 k+1}^{(a)} F_{2 k}^{(a)}+F_{2 k+2}^{(a)} F_{2 k+1}^{(a)} \\
& =\left(F_{2 k}^{(a)}\right)^{2}+F_{2 k+1}^{(a)} F_{2 k}^{(a)}+F_{2 k+2}^{(a)} F_{2 k+1}^{(a)} \\
& =F_{2 k}^{(a)}\left(F_{2 k}^{(a)}+F_{2 k+1}^{(a)}\right)+F_{2 k+2}^{(a)} F_{2 k+1}^{(a)} \\
& =F_{2 k+2}^{(a)}\left(F_{2 k}^{(a)}+F_{2 k+1}^{(a)}\right) \\
& =\left(F_{2 k+2}^{(a)}\right)^{2}
\end{aligned}
$$


which proves (a) for $n=k+1$. For (b), clearly we have

$$
(a-2) \sum_{i=2}^{3} F_{i}^{(a)} F_{i-1}^{(a)}=(a-2)(1+a-1)=(a-2) a=\left(F_{3}^{(a)}\right)^{2} .
$$

Proceeding by induction on $n$, suppose that we have

$$
(a-2) \sum_{i=2}^{2 n+1} F_{i}^{(a)} F_{i-1}^{(a)}=\left(F_{2 n+1}^{(a)}\right)^{2}-1
$$

then,

$$
\begin{aligned}
& (a-2) \sum_{i=2}^{2 k+3} F_{i}^{(a)} F_{i-1}^{(a)} \\
= & (a-2) \sum_{i=2}^{2 k+1} F_{i}^{(a)} F_{i-1}^{(a)}+(a-2)\left(F_{2 k+2}^{(a)} F_{2 k+1}^{(a)}+F_{2 k+3}^{(a)} F_{2 k+2}^{(a)}\right)-1 \\
= & \left(F_{2 k+1}^{(a)}\right)^{2}-1+(a-2)\left(F_{2 k+2}^{(a)} F_{2 k+1}^{(a)}+F_{2 k+3}^{(a)} F_{2 k+2}^{(a)}\right)-1 \\
= & F_{2 k+1}^{(a)}\left(F_{2 k+1}^{(a)}+(a-2) F_{2 k+2}^{(a)}\right)+(a-2) F_{2 k+3}^{(a)} F_{2 k+2}^{(a)}-1 \\
= & F_{2 k+1}^{(a)} F_{2 k+3}^{(a)}+(a-2) F_{2 k+3}^{(a)} F_{2 k+2}^{(a)}-1 \\
= & \left.F_{2 k+3}^{(a)}\left(F_{2 k+1}^{(a)}+(a-2) F_{2 k+2}^{(a)}\right)\right)-1 \\
= & \left(F_{2 k+3}^{(a)}\right)^{2}-1
\end{aligned}
$$

which proves (b) for $n=k+1$.

The following theorem generalizes the well-known fact that the sequence $\left\{\frac{F_{n+k}}{F_{n}}\right\}$ converges to $\left(\frac{1+\sqrt{5}}{2}\right)^{k}$, the $k$-th power of the golden ratio. In the following theorem, we give similar properties for the sequence $\left\{\frac{F_{n+k}^{(a)}}{F_{n}^{(a)}}\right\}$.

Theorem 3.7. (a) $\lim _{n \rightarrow \infty} \frac{F_{n+2 k}^{(a)}}{F_{n}^{(a)}}=\alpha^{k}$.

(b) $\lim _{n \rightarrow \infty} \frac{F_{2 n+2 k+1}^{(a)}}{F_{2 n}^{(a)}}=\alpha^{k}(\alpha-1)$.

(c) $\lim _{n \rightarrow \infty} \frac{F_{2 n+1+2 k+1}^{(a)}}{F_{2 n+1}^{(a)}}=\frac{\alpha^{k+1}}{\alpha-1}$.

Proof. Combining Proposition 3.5 with $|\alpha-1|>1$, we have

$$
\begin{aligned}
\lim _{n \rightarrow \infty} \frac{F_{n+2 k}^{(a)}}{F_{n}^{(a)}} & =\lim _{n \rightarrow \infty} \frac{\frac{1}{(a-2)^{\left[\frac{n+2 k}{2}\right]} \sqrt{a^{2}-4}}\left((\alpha-1)^{n+2 k}-\left(\frac{1}{\alpha}-1\right)^{n+2 k}\right)}{\frac{1}{(a-2)^{\left[\frac{n}{2}\right]} \sqrt{a^{2}-4}}\left((\alpha-1)^{n}-\left(\frac{1}{\alpha}-1\right)^{n}\right)} \\
& =\lim _{n \rightarrow \infty} \frac{\left((\alpha-1)^{n+2 k}-\left(\frac{1}{\alpha}-1\right)^{n+2 k}\right)}{(a-2)^{k}\left((\alpha-1)^{n}-\left(\frac{1}{\alpha}-1\right)^{n}\right)}
\end{aligned}
$$




$$
\begin{aligned}
& =\lim _{n \rightarrow \infty}\left(\frac{(\alpha-1)^{2}}{a-2}\right)^{k} \\
& =\alpha^{k}
\end{aligned}
$$

and hence (a). For (b),

$$
\begin{aligned}
\lim _{n \rightarrow \infty} \frac{F_{2 n+1+2 k}^{(a)}}{F_{2 n}^{(a)}} & =\lim _{n \rightarrow \infty} \frac{\frac{1}{(a-2)^{n+k} \sqrt{a^{2}-4}}\left((\alpha-1)^{2 n+1+2 k}-\left(\frac{1}{\alpha}-1\right)^{2 n+1+2 k}\right)}{\frac{1}{(a-2)^{n} \sqrt{a^{2}-4}}\left((\alpha-1)^{2 n}-\left(\frac{1}{\alpha}-1\right)^{2 n}\right)} \\
& =\lim _{n \rightarrow \infty} \frac{\left((\alpha-1)^{2 n+1+2 k}-\left(\frac{1}{\alpha}-1\right)^{2 n+1+2 k}\right)}{(a-2)^{k}\left((\alpha-1)^{2 n}-\left(\frac{1}{\alpha}-1\right)^{2 n}\right)} \\
& =\lim _{n \rightarrow \infty}\left(\frac{(\alpha-1)^{2}}{a-2}\right)^{k}(\alpha-1) \\
& =\alpha^{k}(\alpha-1) .
\end{aligned}
$$

In a similar manner, we can derive part (c).

Corollary 3.8. (a) $\left(\left(F_{2 j}^{(a)}, F_{2 j+2}^{(a)}\right) \mid\left(F_{2 j+2}^{(a)}, F_{2 j+4}^{(a)}\right)\right)=$ a for all $j \in \mathbb{Z}_{\geq 0}$.

(b) $\left(\left(F_{2 j+1}^{(a)}, F_{2 j+3}^{(a)}\right) \mid\left(F_{2 j+3}^{(a)}, F_{2 j+5}^{(a)}\right)\right)=-(a-2)$ a for all $j \in \mathbb{Z}_{\geq 0}$.

Proof. The part (a) is an immediate consequence of the parts (e) and (f) of Theorem 3.1. Combining the results of the part(a) and Theorem 3.1, we have

$$
\begin{aligned}
& \left(\left(F_{2 j+1}^{(a)}, F_{2 j+3}^{(a)}\right) \mid\left(F_{2 j+3}^{(a)}, F_{2 j+5}^{(a)}\right)\right) \\
= & \left(\left(F_{2 j+2}^{(a)}-F_{2 j}^{(a)}, F_{2 j+4}^{(a)}-F_{2 j+2}^{(a)}\right) \mid\left(F_{2 j+4}^{(a)}-F_{2 j+2}^{(a)}, F_{2 j+6}^{(a)}-F_{2 j+4}^{(a)}\right)\right) \\
= & \left(\left(F_{2 j+2}^{(a)}, F_{2 j+4}^{(a)}\right) \mid\left(F_{2 j+4}^{(a)}, F_{2 j+6}^{(a)}\right)\right)-\left(\left(F_{2 j+2}^{(a)}, F_{2 j+4}^{(a)}\right) \mid\left(F_{2 j+2}^{(a)}, F_{2 j+4}^{(a)}\right)\right) \\
& -\left(\left(F_{2 j}^{(a)}, F_{2 j+2}^{(a)}\right) \mid\left(F_{2 j+4}^{(a)}, F_{2 j+6}^{(a)}\right)\right)+\left(\left(F_{2 j}^{(a)}, F_{2 j+2}^{(a)}\right) \mid\left(F_{2 j+2}^{(a)}, F_{2 j+4}^{(a)}\right)\right) \\
= & a-2-\left(-a^{2}+2\left(a^{2}-1\right)\right)+a \\
= & -(a-2) a,
\end{aligned}
$$

and hence (b).

\section{References}

[1] R. A. Dunlap, The golden ratio and Fibonachi numbers, World Science, 1997.

[2] A. J. Feingold, A hyperbolic GCM Lie algebra and the Fibonachi numbers, Proc. Amer. Math. Soc. 80 (1980), 379-385.

[3] A. F. Horadam, A Generalized the Fibonachi Sequence, Proc. Amer. Math. Monthly. 68 (1961), 455-459.

[4] V. G. Kac, Infinite-Dimensional Lie Algebras, Cambridge University Press, 1990.

[5] S. J. Kang and D. J. Melville, Rank 2 Symmetric Hyperbolic Kac-Moody Algebras, Nagoya Math. J. 140 (1995), 41-75. 
[6] J. Moragado, Some remark on an identy of Catalan concerning the Fibonachi numbers, Portugaliae Math. Soc. 39 (1980), 341-348.

[7] K. S. Rao, Some Propertities of Fibonachi numbers, Amer. Math. Monthly. 60 (1953), $680-684$

Department of Mathematics

SOONGSil UNIVERSITY

SEOUl 156-743, KoreA

E-mail address: yokim@ssu.ac.kr 